\title{
Theoretical Issues on LTI Systems That Preserve Signal Richness
}

\author{
Borching Su, Student Member, IEEE, and P. P. Vaidyanathan, Fellow, IEEE
}

\begin{abstract}
In this paper, theoretical issues about linear time invariant (LTI) systems that preserve signal richness are explored. This paper considers two particular definitions of signal richness and finds the necessary and sufficient conditions under which an LTI system preserves the richness property. Several examples are presented to clarify the issues involved in the problem. Paraunitary (PU) and unimodular matrices can be shown not to preserve richness unless they are constant matrices (or a delayed version in the $\mathrm{PU}$ case). Some richness preserving properties of cascaded systems are also investigated. A structured proof of the necessary and sufficient conditions is presented. The relationship between persistent excitation (PE) and the proposed definitions of richness is also described.
\end{abstract}

Index Terms-Blind identification, full rank, persistent excitation, richness.

\section{INTRODUCTION}

$\mathbf{V}$ ECTORIZED signals are often considered to be "rich" if they satisfy certain fullness properties appropriate for an application under discussion. In some applications, a sequence of $M \times 1$ vectors $\mathbf{x}(n), n \geq 0$ is said to be rich or rank-rich if the matrix

$$
\left[\begin{array}{llll}
\mathbf{x}(0) & \mathbf{x}(1) & \cdots & \mathbf{x}\left(K_{x}\right)
\end{array}\right]
$$

has rank $M$ for sufficiently large $K_{x}$ [2]. This property is important, for example, when we try to identify an unknown communication channel from output measurements alone using filter bank precoders [5]. Now, signals are sometimes preconditioned by linear transformations before they are used in such an application [6]. This leads us to explore the conditions under which the linear precoders will preserve richness of the vectorized signals. This fundamental mathematical problem, rather than the applications, will be the focus of this paper and will be explored in depth. For further details of these applications, the reader should refer to [5] and [7].

Let the linear time invariant (LTI) system be characterized by the $M \times M$ polynomial matrix

$$
\mathbf{H}(z)=\sum_{k=0}^{N} \mathbf{h}(k) z^{-k}
$$

Manuscript received January 20, 2005; revised April 22, 2005. This work is supported in parts by the NSF Grant CCF-0428326 and the Moore Fellowship of the California Institute of Technology. The associate editor coordinating the review of this manuscript and approving it for publication was Dr. Behrouz Farhang-Boroujeny.

The authors are with the Department of Electrical Engineering, California Institute of Technology, Pasadena, CA 91125 USA.

Digital Object Identifier 10.1109/TSP.2005.863043 so that the output of the system is

$$
\mathbf{y}(n)=\sum_{k=0}^{N} \mathbf{h}(k) \mathbf{x}(n-k) .
$$

We say the system $\mathbf{H}(z)$ is richness preserving (RP) if for any rank-rich input $\mathbf{x}(n)$, the output $\mathbf{y}(n)$ is also rank-rich.

This paper is organized as follows. In Section II, the first definition of richness will be given and several examples will be presented to clarify the issue. A set of necessary and sufficient conditions will be presented in Section III. In Section IV, we will explore more properties of richness preserving systems, including cascaded systems, and enriching systems, and we will also show that paraunitary (PU) matrices and unimodular matrices cannot satisfy the necessary conditions unless they are constant matrices (with a possible delay in the PU case). In Section V, a strict definition of richness and the necessary and sufficient conditions on LTI systems that preserve richness according to this definition will be given. The proof of the main theorems will be given in Section VI. In Section VII we will connect the relationship between strict richness defined in Section V and persistent excitation (PE) in the literature on control theory [9]-[12]. Conclusions and open issues are presented in Section VIII. Some parts of this paper have been presented at conferences [2], [3], and [8].

\section{A. Notations}

Boldfaced lower case letters represent column vectors, and boldfaced upper case letters are reserved for matrices. Superscripts as in $\mathbf{A}^{T}$ and $\mathbf{A}^{\dagger}$ denote the transpose and transpose-conjugate operations, respectively, of a matrix or a vector. $\widetilde{\mathbf{H}}(z)$ represents $\mathbf{H}^{\dagger}\left(1 / z^{*}\right)$, where superscript “*” denotes complex conjugate. $[\mathbf{v}]_{i}$ denotes the $i$ th element of vector $\mathbf{v}$, and $\mathbf{e}_{i}$ denotes the $i$ th vector of the standard basis of $\mathbb{C}^{M}$. All the vectors and matrices in this context are complex-valued.

\section{FORMULATION AND EXAMPLES}

Definition 1: A sequence of $M \times 1$ vectors $\mathbf{x}(n), n \geq 0$ is said to be rich if there exists an integer $K_{x}$ such that the matrix

$$
\left[\begin{array}{llll}
\mathbf{x}(0) & \mathbf{x}(1) & \cdots & \left.\mathbf{x}\left(K_{x}\right)\right)
\end{array}\right]
$$

has rank $M$.

Consider an LTI finite-impulse-response (FIR) causal system $\mathbf{H}(z)=\sum_{k=0}^{N} \mathbf{h}(k) z^{-k}$. Then the output of this system is rich if there exists an integer $K_{y}$ such that

$$
\mathcal{Y}=\left[\begin{array}{llll}
\mathbf{y}(0) & \mathbf{y}(1) & \cdots & \mathbf{y}\left(K_{y}\right)
\end{array}\right]
$$


has rank $M$. Note that $\mathcal{Y}=\mathcal{H} \mathcal{X}$, where

and

$$
\mathcal{H}=[\mathbf{h}(0) \mathbf{h}(1) \cdots \mathbf{h}(N)]
$$

$$
\mathcal{X}=\left[\begin{array}{ccccc}
\mathrm{x}(0) & \mathrm{x}(1) & \mathrm{x}(2) & \cdots & \mathrm{x}\left(K_{y}\right) \\
\mathbf{0} & \mathrm{x}(0) & \mathrm{x}(1) & \cdots & \mathrm{x}\left(K_{y}-1\right) \\
\mathbf{0} & \mathbf{0} & \mathrm{x}(0) & \cdots & \mathrm{x}\left(K_{y}-2\right) \\
\vdots & \vdots & & \ddots & \vdots \\
\mathbf{0} & \mathbf{0} & \mathbf{0} & \cdots & \mathrm{x}\left(K_{y}-N\right)
\end{array}\right]
$$

The matrix $\mathcal{H}$ has size $M \times M(N+1)$. With $\rho_{y}, \rho_{h}$, and $\rho_{x}$ denoting the ranks of $\mathcal{Y}, \mathcal{H}$, and $\mathcal{X}$, respectively, we have from Sylvester's inequality [1]

$$
\rho_{h}+\rho_{x}-M(N+1) \leq \rho_{y} \leq \min \left(\rho_{h}, \rho_{x}\right) .
$$

Observe that if the output matrix $\mathcal{Y}$ has to have rank $M$, it is necessary that the filter matrix $\mathcal{H}$ have rank $M$. For example, if one of the $\mathbf{h}(n)$ 's has rank $M$, this is satisfied. We will produce examples to demonstrate that this necessary condition is in fact not sufficient. In fact the examples also show that many standard systems such as unimodular and PU matrices do not preserve richness!

\section{A. Examples That Do Not Preserve Richness}

Example 1: To demonstrate that the rank- $M$ property of the filter matrix $\mathcal{H}$ is not sufficient, consider the following example with $M=2$ :

$$
\mathbf{H}(z)=\left[\begin{array}{ll}
1 & 1 \\
1 & 1
\end{array}\right]+z^{-1}\left[\begin{array}{cc}
1 & -1 \\
-1 & 1
\end{array}\right] .
$$

Then

$$
\mathcal{H}=\left[\begin{array}{cccc}
1 & 1 & 1 & -1 \\
1 & 1 & -1 & 1
\end{array}\right]
$$

and has rank $M=2$. Suppose the input signal is

$$
\mathbf{x}(0)=\left[\begin{array}{c}
1 \\
-1
\end{array}\right], \quad \mathbf{x}(1)=\left[\begin{array}{l}
-1 \\
-1
\end{array}\right]
$$

with $\mathbf{x}(n)=\mathbf{0}$ otherwise. Clearly, this input is rich because

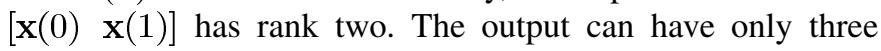
nonzero samples so that the largest output matrix we need to look at is

$$
[\mathbf{y}(0) \mathbf{y}(1) \mathbf{y}(2)]=\left[\begin{array}{ll}
\mathbf{h}(0) & \mathbf{h}(1)
\end{array}\right]\left[\begin{array}{ccc}
\mathbf{x}(0) & \mathbf{x}(1) & \mathbf{0} \\
\mathbf{0} & \mathbf{x}(0) & \mathbf{x}(1)
\end{array}\right] .
$$

We have

$$
\begin{aligned}
{[\mathbf{y}(0) \mathbf{y}(1) \mathbf{y}(2)] } & =\left[\begin{array}{cccc}
1 & 1 & 1 & -1 \\
1 & 1 & -1 & 1
\end{array}\right]\left[\begin{array}{ccc}
1 & -1 & 0 \\
-1 & -1 & 0 \\
0 & 1 & -1 \\
0 & -1 & -1
\end{array}\right] \\
& =\left[\begin{array}{ccc}
0 & 0 & 0 \\
0 & -4 & 0
\end{array}\right]
\end{aligned}
$$

which shows that the output matrix has rank one. Thus, richness of the input is not preserved at the output even though the matrix $\mathcal{H}$ has full rank $M$. In this example $\mathbf{H}(z)$ happens to be a paraunitary (PU) matrix [4], that is, it satisfies $\widetilde{\mathbf{H}}(z) \mathbf{H}(z)=d \mathbf{I}$ for some positive $d$. Thus, PU matrices do not necessarily preserve richness.
Example 2: Consider again $M=2$ and let

$$
\mathbf{H}(z)=\left[\begin{array}{cc}
1+z^{-1} & -z^{-1} \\
z^{-1} & 1-z^{-1}
\end{array}\right]=\left[\begin{array}{ll}
1 & 0 \\
0 & 1
\end{array}\right]+z^{-1}\left[\begin{array}{cc}
1 & -1 \\
1 & -1
\end{array}\right] \text {. }
$$

Then

$$
\mathcal{H}=\left[\begin{array}{llll}
1 & 0 & 1 & -1 \\
0 & 1 & 1 & -1
\end{array}\right]
$$

and has rank $M=2$. Consider the input

$$
\mathbf{x}(0)=\left[\begin{array}{l}
0 \\
1
\end{array}\right], \quad \mathbf{x}(1)=\left[\begin{array}{l}
1 \\
1
\end{array}\right]
$$

with $\mathbf{x}(n)=0$ otherwise. Then the output matrix is

$$
\begin{aligned}
{[\mathbf{y}(0) \mathbf{y}(1) \mathbf{y}(2)] } & =\left[\begin{array}{llll}
1 & 0 & 1 & -1 \\
0 & 1 & 1 & -1
\end{array}\right]\left[\begin{array}{lll}
0 & 1 & 0 \\
1 & 1 & 0 \\
0 & 0 & 1 \\
0 & 1 & 1
\end{array}\right] \\
& =\left[\begin{array}{lll}
0 & 0 & 0 \\
1 & 0 & 0
\end{array}\right]
\end{aligned}
$$

which has rank one. Again richness of the input is not preserved at the output, though $\mathcal{H}$ has full rank $M$. In this example, $\mathbf{H}(z)$ happens to be a unimodular matrix [4], that is, $\operatorname{det} \mathbf{H}(z)=1$ for all $z$ so that its inverse is an FIR matrix as well. The example shows that unimodular matrices do not necessarily preserve richness.

\section{B. Examples That Preserve Richness}

If $\mathbf{H}(z)$ is an invertible memoryless system (i.e., a constant nonsingular matrix), it obviously preserves richness since multiplication with a nonsingular matrix does not change the rank of a matrix. A generalization of this special case has been found in [2] to be sufficient to preserve richness.

Example 3: An $N$ th order FIR system of the form

$$
\mathbf{H}(z)=\mathbf{A}\left(g_{0}+g_{1} z^{-1}+\cdots+g_{N} z^{-N}\right)
$$

preserves richness if $\mathbf{A}$ is a nonsingular matrix and $g_{0} \neq 0$. To see this, suppose there exists a rich input sequence $\mathbf{x}(n)$ such that the output $\mathbf{y}(n)$ is not rich. Then there exists a vector $\mathbf{v} \neq \mathbf{0}$ such that

$$
\mathbf{v}^{\dagger} \mathbf{y}(n)=\mathbf{0}, \quad \forall n
$$

For $n=0$, we have $g_{0} \mathbf{v}^{\dagger} \mathbf{A x}(0)=\mathbf{0}$. Since $g_{0} \neq 0$, we obtain $\mathbf{w}^{\dagger} \mathbf{x}(0)=\mathbf{0}$, where $\mathbf{w}$, defined as $\mathbf{A}^{\dagger} \mathbf{v}$, is also a nonzero vector. For $n=1$, we have $g_{0} \mathbf{v}^{\dagger} \mathbf{x}(1)+g_{1} \mathbf{v}^{\dagger} \mathbf{A} \mathbf{x}(0)=\mathbf{0}$, which implies $\mathbf{w}^{\dagger} \mathbf{x}(1)=\mathbf{0}$ because the second term is zero. For $n=2$, we have $g_{0} \mathbf{v}^{\dagger} \mathbf{x}(2)+g_{1} \mathbf{v}^{\dagger} \mathbf{A} \mathbf{x}(1)+g_{2} \mathbf{v}^{\dagger} \mathbf{A} \mathbf{x}(0)=\mathbf{0}$, and this implies $\mathbf{w}^{\dagger} \mathbf{x}(2)=\mathbf{0}$ since the last two terms are zero. Proceeding like this, we see that $\mathbf{w}^{\dagger} \mathbf{x}(n)=\mathbf{0}$ for all $n$, contradicting the assumption that $\mathbf{x}(n)$ is rich.

Example 4: An RP example that does not have the form of the previous example is [2]

$$
\mathbf{H}(z)=\left[\begin{array}{ll}
1 & a \\
0 & 0
\end{array}\right]+z^{-1}\left[\begin{array}{ll}
0 & 0 \\
1 & a
\end{array}\right],
$$

where $a$ is an arbitrary number. 
To see this, assume the input $\mathbf{x}(n)$ is rich. Denote $x_{n}=$ $\left[\begin{array}{ll}1 & a\end{array}\right] \mathbf{x}(n)$, then $x_{n}$ cannot be zero for all $n$ since $\mathbf{x}(n)$ is rich. Now we have

$$
\mathbf{y}(0)=\left[\begin{array}{ll}
1 & a \\
0 & 0
\end{array}\right] \mathbf{x}(0)=\left[\begin{array}{c}
x_{0} \\
0
\end{array}\right]
$$

and

$$
\mathbf{y}(n)=\left[\begin{array}{ll}
1 & a \\
0 & 0
\end{array}\right] \mathbf{x}(n)+\left[\begin{array}{ll}
0 & 0 \\
1 & a
\end{array}\right] \mathbf{x}(n-1)=\left[\begin{array}{c}
x_{n} \\
x_{n-1}
\end{array}\right]
$$

for $n \geq 1$. Suppose $x_{k}$ is the first nonzero number in the sequence $\left\{x_{n}\right\}$, then

$$
\left[\begin{array}{ll}
\mathbf{y}(k) & \mathbf{y}(k+1)
\end{array}\right]=\left[\begin{array}{cc}
x_{k} & x_{k+1} \\
0 & x_{k}
\end{array}\right]
$$

is a full-rank matrix. So $\mathbf{y}(n)$ is rich for any rich input $\mathbf{x}(n)$.

\section{MAIN THEOREM}

In this section, we will describe the necessary and sufficient conditions for an LTI system to preserve richness. The proof of the theorem will be given in Section VI.

Theorem 1: An $N$ th order, $M \times M$ polynomial matrix

$$
\mathbf{H}(z)=\sum_{k=0}^{N} \mathbf{h}(k) z^{-k}
$$

is an RP LTI system if and only if either one of the following conditions is true:

a) there exist a nonsingular $M \times M$ matrix $\mathbf{A}$ and constants $g_{0}, g_{1}, \cdots, g_{N}$ of which at least one is nonzero such that $\mathbf{h}(k)=g_{k} \mathbf{A}$

b) there exist a nonzero row vector $\mathbf{v}^{\dagger}$ and a set of column vectors $\mathbf{a}_{0}, \mathbf{a}_{1}, \cdots, \mathbf{a}_{N}$ such that $\mathbf{h}(k)=\mathbf{a}_{k} \mathbf{v}^{\dagger}$ for any $k$, and $\left[\begin{array}{llll}\mathbf{a}_{0} & \mathbf{a}_{1} & \cdots & \mathbf{a}_{N}\end{array}\right]$ has full rank $M$.

It is obvious that conditions a) and b) cannot be satisfied at the same time. Hence, we can say there are two types of RP matrices, namely, Type A and Type B, according to the statement of Theorem 1. Examples 3 and 4 in the previous section serve as special cases for Type A and Type B RP matrices, respectively.

For a Type A matrix, each nonzero coefficient matrix is nonsingular. For Type B matrices, each nonzero coefficient matrix has unit rank. There are no other types of examples! Notice in particular that the order $N$ and the size $M$ of a Type B matrix must satisfy $N \geq M-1$ to meet the full rank criterion of $\left[\mathbf{a}_{0} \mathbf{a}_{1}, \cdots \mathbf{a}_{N}\right]$.

The rank of each nonzero coefficient matrix of an RP matrix is always the same, and we call it the coefficient rank. In addition, the coefficient rank of an RP system can only be either unity or full. For an RP system where the first coefficient matrix is nonsingular, a useful corollary of Theorem 1 is as follows.

Corollary 1: Consider the $N$ th order, $M \times M$ FIR system $\mathbf{H}(z)=\sum_{k=0}^{N} \mathbf{h}(k) z^{-k}$ and assume $\mathbf{h}(0)$ is nonsingular. Then $\mathbf{H}(z)$ is RP if and only if there exist a nonsingular $M \times M$ matrix $\mathbf{A}$ and constants $g_{0}, g_{1}, \ldots, g_{N}$, where $g_{0} \neq 0$ such that $\mathbf{h}(k)=g_{k} \mathbf{A}$.
When the first coefficient matrix $\mathbf{h}(0)$ of a RP system is singular but nonzero, it must be a Type B RP system, as stated in the following corollary.

Corollary 2: Consider the $N$ th order, $M \times M$ FIR system $\mathbf{H}(z)=\sum_{k=0}^{N} \mathbf{h}(k) z^{-k}$ with size $M \times M$ and assume $\mathbf{h}(0) \neq$ $\mathbf{0}$ is singular. Then $\mathbf{H}(z)$ is RP if and only if there exist a nonzero row vector $\mathbf{v}^{\dagger}$ and $N+1$ column vectors $\mathbf{a}_{0}, \mathbf{a}_{1}, \cdots \mathbf{a}_{N}$ such that $\mathbf{h}(n)=\mathbf{a}_{n} \mathbf{v}^{\dagger},\left[\begin{array}{llll}\mathbf{a}_{0} & \mathbf{a}_{1} & \cdots & \mathbf{a}_{N}\end{array}\right]$ has full rank, and $\mathbf{a}_{0} \neq \mathbf{0}$.

The proofs of the preceding two corollaries will be automatically covered when we prove Theorem 1 in Section VI. In these corollaries, we have not considered the case where $\mathbf{h}(0)=\mathbf{0}$. If this is true, however, $\mathbf{H}(z)$ is simply a delayed version of another LTI system whose first coefficient is nonzero. Since $\mathbf{H}(z)$ is RP if and only if $z^{-m} \mathbf{H}(z)$ is RP for any $m$, the assumption $\mathbf{h}(0) \neq \mathbf{0}$ is not a loss of generality.

\section{A. Proof of a Special Case}

We will give the proof of a special case of Corollary 1 where we assume $\mathbf{h}(0)$ is nonsingular. Although the proof of this special case will be definitely covered when we prove Theorem 1 in Section VI, the reader might find this insightful.

Special Case of Corollary 1: Consider the first order, $2 \times 2$ FIR system

$$
\mathbf{H}(z)=\mathbf{h}(0)+z^{-1} \mathbf{h}(1)
$$

and assume $\mathbf{h}(0)$ is nonsingular. Then $\mathbf{H}(z)$ is $\mathrm{RP}$ if and only if $\mathbf{h}(1)=\rho \mathbf{h}(0)$ for some sclar constant $\rho$.

Proof: The proof of sufficiency is self-evident in view of Example 3. For necessity, since $\mathbf{h}(0)$ is nonsingular, we can write $\mathbf{H}(z)=\mathbf{h}(0)\left(\mathbf{I}+\mathbf{B} z^{-1}\right)$. The nonsingular factor $\mathbf{h}(0)$ does not affect the rank of the output matrix. So $\mathbf{H}(z)$ is RP if and only if $\left(\mathbf{I}+\mathbf{B} z^{-1}\right)$, which has the form

$$
\mathbf{G}(z)=\mathbf{I}+z^{-1}\left[\begin{array}{ll}
a & b \\
c & d
\end{array}\right]
$$

preserves richness. Consider the input

$$
\mathbf{x}(0)=\left[\begin{array}{l}
0 \\
1
\end{array}\right], \quad \mathbf{x}(1)=\left[\begin{array}{c}
-b \\
a
\end{array}\right]
$$

with $\mathbf{x}(n)=\mathbf{0}$ otherwise. This produces the output

$$
\mathbf{y}(0)=\left[\begin{array}{l}
0 \\
1
\end{array}\right], \quad \mathbf{y}(1)=\left[\begin{array}{c}
0 \\
a+d
\end{array}\right], \quad \mathbf{y}(2)=\left[\begin{array}{c}
0 \\
a d-b c
\end{array}\right]
$$

and $\mathbf{y}(n)=\mathbf{0}$ otherwise. We see that if $b \neq 0$, then $[\mathbf{x}(0) \mathbf{x}(1)]$ has rank two, and hence the input is rich while the output $\mathbf{y}(n)$ is not. So $b=0$ is a necessary condition for richness preservation. A slight variation of this construction shows that $c=0$ is necessary as well. Thus, in order to preserve richness $\mathbf{G}(z)$ has to be of the form

$$
\mathbf{G}(z)=\mathbf{I}+z^{-1}\left[\begin{array}{ll}
a & 0 \\
0 & d
\end{array}\right] .
$$

If we now choose the input

$$
\mathbf{x}(0)=\left[\begin{array}{l}
1 \\
1
\end{array}\right], \quad \mathbf{x}(1)=\left[\begin{array}{l}
d \\
a
\end{array}\right]
$$


with $\mathbf{x}(n)=\mathbf{0}$ otherwise, then

$$
\mathbf{y}(0)=\left[\begin{array}{l}
1 \\
1
\end{array}\right], \quad \mathbf{y}(1)=\left[\begin{array}{l}
a+d \\
a+d
\end{array}\right], \quad \mathbf{y}(2)=\left[\begin{array}{l}
a d \\
a d
\end{array}\right]
$$

with $\mathbf{y}(n)=\mathbf{0}$ otherwise. If $a \neq d$, then the input is rich, whereas the output is not. This shows that $a=d$ is a necessary condition. Thus, $\mathbf{G}(z)=\mathbf{I}+\rho \mathbf{I} z^{-1}$, so $\mathbf{h}(1)=\rho \mathbf{h}(0)$ indeed.

\section{Properties of Richness-PRESERVING Systems}

\section{A. Cascaded Systems}

In this subsection, we are interested in richness-preserving properties of cascaded systems. It is obvious that the product of RP systems is also RP. We will show that the product is Type A RP if all the subsystems are Type A RP, and it would be Type B $\mathrm{RP}$ if any of them is Type B RP.

Theorem 2: If $\mathbf{A}_{1}(z), \mathbf{A}_{2}(z)$ are Type A RP matrices and $\mathbf{B}_{1}(z), \mathbf{B}_{2}(z)$ are Type B RP matrices, then the following is found:

1) $\mathbf{A}_{1}(z) \mathbf{A}_{2}(z)$ is a Type A RP matrix;

2) $\mathbf{A}_{1}(z) \mathbf{B}_{1}(z)$ is a Type B RP matrix;

3) $\mathbf{B}_{1}(z) \mathbf{A}_{1}(z)$ is a Type B RP matrix;

4) $\mathbf{B}_{1}(z) \mathbf{B}_{2}(z)$ is a Type $\mathrm{B} \mathrm{RP}$ matrix.

Proof: $\mathbf{A}_{1}(z)=g_{1}(z) \mathbf{A}_{1}, \mathbf{A}_{2}(z)=g_{2}(z) \mathbf{A}_{2}$, where $\mathbf{A}_{1}, \mathbf{A}_{2}$ are invertible constant $M \times M$ matrices and $g_{1}(z), g_{2}(z)$ are nonzero polynomials in $z^{-1}$. Then the product $\mathbf{A}_{1} \mathbf{A}_{2}=g_{1}(z) g_{2}(z) \mathbf{A}_{1} \mathbf{A}_{2}$ is clearly a Type A RP matrix. Furthermore, let $\mathbf{B}_{1}(z)=\sum_{k=0}^{N_{1}} \mathbf{a}_{k} \mathbf{v}_{1}^{\dagger} z^{-k}$ and $\mathbf{B}_{2}(z)=\sum_{k=0}^{N_{2}} \mathbf{b}_{k} \mathbf{v}_{2}^{\dagger} z^{-k}$, then $\mathbf{A}_{1}(z) \mathbf{B}_{1}(z)=g_{1}(z) \mathbf{A}_{1} \sum_{k=0}^{N_{1}} \mathbf{a}_{k} \mathbf{v}_{1}^{\dagger} z^{-k}=$ $\sum_{l=0}^{N} g_{l} z^{-l} \sum_{k=0}^{N_{1}} \mathbf{a}_{k}^{\prime} z^{-k} \mathbf{v}_{1}^{\dagger}=\sum_{k=0}^{N_{1}+N} \mathbf{a}_{k}^{\prime \prime} z^{-k} \mathbf{v}_{1}^{\dagger}$ is Type B RP since both $\mathbf{a}_{k}^{\prime}$ 's and $\mathbf{a}_{k}^{\prime \prime}$ 's still span a full dimensional space. In addition, $\mathbf{B}_{1}(z) \mathbf{A}_{1}(z)=\left(\sum_{k=0}^{N_{1}} \mathbf{a}_{k} z^{-k}\right) g_{1}(z) \mathbf{v}_{1}^{\dagger} \mathbf{A}_{1}=$ $\left(\sum_{k=0}^{N_{1}+N} \mathbf{a}_{k}^{\prime} z^{-k}\right) \mathbf{v}^{\prime \dagger}$ is also Type B RP. Finally $\mathbf{B}_{1}(z) \mathbf{B}_{2}(z)=$ $\left(\sum_{k=0}^{N_{1}} \mathbf{a}_{k} \mathbf{v}_{1}^{\dagger} z^{-k}\right)\left(\sum_{l=0}^{N_{2}} \mathbf{b}_{l} \mathbf{v}_{2}^{\dagger} z^{-l}\right)=\sum_{k=0}^{N_{1}+N_{2}} \mathbf{a}_{k}^{\prime} \mathbf{v}_{2}^{\dagger} z^{-k}$ is also a Type B RP matrix.

If some of the subsystems are non-RP, however, it does not imply the whole system is non-RP. A trivial example is a cascade of the unimodular matrix in Example 2 with its inverse, which is also causal and unimodular. Since the product is identity it preserves richness. But both of the factors in the product are non-RP systems. In fact, for a cascaded system to be RP, although it is sufficient that all the subsystems are RP, this is not necessary. An interesting question that comes up here is this: If $\mathbf{F}(z) \mathbf{H}(z) \mathbf{G}(z)$ is a richness-preserving system and both $\mathbf{F}(z)$ and $\mathbf{G}(z)$ are $\mathrm{RP}$, is $\mathbf{H}(z)$ also RP? The answer depends on the types of $\mathbf{F}(z)$ and $\mathbf{G}(z)$ and is given in the following two theorems.

Theorem 3: Suppose $\mathbf{A}(z)$ is a Type A RP matrix. Then the statement " $\mathbf{A}(z) \mathbf{H}(z)$ is RP" implies that $\mathbf{H}(z)$ is RP. Similarly the statement " $\mathbf{H}(z) \mathbf{A}(z)$ is $\mathrm{RP}$ " also implies that $\mathbf{H}(z)$ is $\mathrm{RP}$.

This theorem states that if a Type A RP system is going to connect with another system, the resulting cascaded system is $\mathrm{RP}$ only when the new system is also RP. On the contrary, Type B RP systems do not have this property. We can see this in the following examples.
Example 5: Let $\mathbf{B}(z)=\left[\begin{array}{cc}1 & 0 \\ z^{-1} & 0\end{array}\right]$, which is a Type B RP system and

$$
\mathbf{H}_{1}(z)=\left[\begin{array}{ll}
1 & 1 \\
0 & 0
\end{array}\right]
$$

then

$$
\mathbf{B}(z) \mathbf{H}_{1}(z)=\left[\begin{array}{cc}
1 & 0 \\
z^{-1} & 0
\end{array}\right]\left[\begin{array}{ll}
1 & 1 \\
0 & 0
\end{array}\right]=\left[\begin{array}{cc}
1 & 1 \\
z^{-1} & z^{-1}
\end{array}\right]
$$

is $\mathrm{RP}$ although $\mathbf{H}_{1}(z)$ is not RP. On the other hand, let

$$
\mathbf{H}_{2}(z)=\left[\begin{array}{cc}
1+z^{-1} & -1 \\
z^{-1} & 0
\end{array}\right]
$$

We have that

$$
\mathbf{H}_{2}(z) \mathbf{B}(z)=\left[\begin{array}{cc}
1+z^{-1} & -1 \\
z^{-1} & 0
\end{array}\right]\left[\begin{array}{cc}
1 & 0 \\
z^{-1} & 0
\end{array}\right]=\left[\begin{array}{cc}
1 & 0 \\
z^{-1} & 0
\end{array}\right]
$$

is also $\mathrm{RP}$ while $\mathbf{H}_{2}(z)$ is not.

Actually, for any given Type B RP system, we can always find a nonrich system such that the product of the two systems is RP, as stated in the following theorem.

Theorem 4: If $\mathbf{B}(z)$ is a Type B RP matrix, then there exist non-RP systems $\mathbf{H}_{1}(z)$ and $\mathbf{H}_{2}(z)$ such that $\mathbf{B}(z) \mathbf{H}_{1}(z)$ and $\mathbf{H}_{2}(z) \mathbf{B}(z)$ are both RP.

The proofs of these two theorems require some lemmas which will be introduced in the following two subsections. The proofs will be given in Section IV-D.

\section{B. Enriching Systems}

We can define a system to be enriching if there exists a nonrich input such that the output of the system is rich. An enriching system, when following a non-RP system, could possibly make the overall system become RP again. We will show that Type A RP matrices are not enriching while all Type B RP matrices are enriching.

Lemma 1: If $\mathbf{A}(z)$ is a Type A RP system, then the input $\mathbf{x}(n)$ is rich if and only if the output $\mathbf{y}(n)$ is rich.

Proof: Obviously, if $\mathbf{x}(n)$ is rich, then so is $\mathbf{y}(n)$. Now suppose $\mathbf{x}(n)$ is nonrich, we need to show that $\mathbf{y}(n)$ is also nonrich. Let

$$
\mathbf{A}(z)=\sum_{k=0}^{N} g_{k} \mathbf{A} z^{-k}
$$

where $\mathbf{A}$ is an invertible constant matrix. We have

$$
\mathbf{y}(n)=\sum_{k=0}^{N} g_{k} \mathbf{A} \mathbf{x}(n-k)=\sum_{k=0}^{N} g_{k} \mathbf{x}^{\prime}(n-k)
$$

where $\mathbf{x}^{\prime}(n)=\mathbf{A} \mathbf{x}(n)$ is also nonrich since $\mathbf{A}$ does not change the rank of a signal. This implies $\mathbf{y}(n)$, a linear combination of $\mathbf{x}^{\prime}(k)$, is also nonrich.

This lemma states that Type A RP systems are never enriching. Type-B RP systems, on the contrary, can be shown to be enriching. An example of this is when

$$
\mathbf{H}(z)=\left[\begin{array}{ll}
1 & a \\
0 & 0
\end{array}\right]+z^{-1}\left[\begin{array}{ll}
0 & 0 \\
1 & a
\end{array}\right]
$$

and we let the input $\mathrm{x}(n)=\left[\begin{array}{ll}1 & 0\end{array}\right]^{T}$ for all $n$, which is obviously nonrich. Then we obtain $\mathbf{y}(0)=\left[\begin{array}{ll}1 & 0\end{array}\right]^{T}$ and $\mathbf{y}(1)=\left[\begin{array}{ll}1 & 1\end{array}\right]^{T}$, 
implying the output $\mathbf{y}(n)$ is rich, and hence $\mathbf{H}(z)$ is enriching. More generally we have the following.

Lemma 2: If $\mathbf{B}(z)$ is a Type B RP system, then there exists a nonrich input $\mathbf{x}(n)$ such that the output is rich.

Proof: For $\mathbf{B}(z)=\sum_{k=0}^{N} \mathbf{a}_{k} \mathbf{v}^{\dagger} z^{-k}$, let $\mathbf{x}(n)=\mathbf{v} /\|\mathbf{v}\|^{2}$ for all $n$, which is obviously nonrich. Then it can be shown that $\mathbf{y}(n)=\sum_{k=0}^{n} \mathbf{a}_{k}$ for $0 \leq n \leq N$. So we have

$$
[\mathbf{y}(0) \mathbf{y}(1) \cdots \mathbf{y}(N)]=\left[\mathbf{a}_{0} \mathbf{a}_{1} \cdots \mathbf{a}_{N}\right]\left[\begin{array}{cccc}
1 & 1 & \cdots & 1 \\
0 & 1 & \cdots & 1 \\
\vdots & \ddots & \ddots & \vdots \\
0 & \cdots & 0 & 1
\end{array}\right]
$$

which implies the matrix $[\mathbf{y}(0) \mathbf{y}(1) \cdots \mathbf{y}(N)]$ has full rank since it is the product of two full-rank matrices. So $\mathbf{y}(n)$ is rich, and hence any Type B RP system is enriching.

\section{Restriction on Output Range}

Consider the cascaded system $\mathbf{F}(z) \mathbf{G}(z)$. If rich signals which are rendered nonrich by $\mathbf{F}(z)$ can never be produced as outputs of $\mathbf{G}(z)$, then the product $\mathbf{F}(z) \mathbf{G}(z)$ can be RP. We will show that a Type A RP system $\mathbf{G}(z)$ can produce any output if the first coefficient matrix $\mathbf{g}(0)$ is nonzero. For any Type B RP system, on the contrary, we can always find an output that it cannot generate.

Lemma 3: If $\mathbf{A}(z)$ is a Type A RP matrix and the first coefficient matrix $\mathbf{h}(0)$ is nonzero, then for any output sequence $\mathbf{y}(n)$, there exists unique $\mathbf{x}(n)$ such that the output of $\mathbf{A}(z)$ is $\mathbf{y}(n)$.

Proof: For any $n \geq 0$ we have

$$
\mathbf{y}(n)=\sum_{k=0}^{N} g_{k} \mathbf{A} \mathbf{x}(n-k)
$$

where we assume $\mathbf{x}(n)=\mathbf{0}$ for all $n<0$. This implies

$$
\mathbf{A}^{-1} \mathbf{y}(n)=g_{0} \mathbf{x}(n)+\sum_{k=1}^{N} g_{1} \mathbf{x}(n-k)
$$

and hence

$$
\mathbf{x}(n)=\frac{1}{g_{0}}\left[\mathbf{A}^{-1} \mathbf{y}(n)-\sum_{k=1}^{N} g_{1} \mathbf{x}(n-k)\right]
$$

can be uniquely decided for any $n \geq 0$, given any output signal $\mathbf{y}(n)$.

Lemma 4: If $\mathbf{B}(z)$ is a Type B RP matrix, then there exists $\mathbf{y}(n)$ that cannot be output of $\mathbf{B}(z)$.

Proof: Suppose

$$
\mathbf{B}(z)=\sum_{k=0}^{N} \mathbf{a}_{k} \mathbf{v}^{\dagger} z^{-k} .
$$

Then for any input $\mathbf{x}(n)$, we have $\mathbf{y}(0)=\mathbf{a}_{0} \mathbf{v}^{\dagger} \mathbf{x}(0)$, which is confined to be a scalar multiple of $\mathbf{a}_{0}$. So $\mathbf{B}(z)$ cannot produce output $\mathbf{y}(n)$ where $\mathbf{y}(0)$ is not proportional to $\mathbf{a}_{0}$.

\section{Proof of Theorems 3 and 4}

Proof of Theorem 3: First let $\mathbf{x}(n)$ be the input to $\mathbf{H}(z), \mathbf{x}_{1}(n)$ be the output of $\mathbf{H}(z)$ and the input of $\mathbf{A}(z)$, and $\mathbf{y}(n)$ be the output $\mathbf{A}(z)$. Suppose $\mathbf{H}(z)$ is not RP but $\mathbf{A}(z) \mathbf{H}(z)$ is RP. Then there exists $\mathbf{x}(n)$ such that $\mathbf{x}_{1}(n)$ is nonrich. From Lemma 1 we know $\mathbf{y}(n)$ is also nonrich. Hence, the system $\mathbf{A}(z) \mathbf{H}(z)$ is not RP, contradicting the assumption. So $\mathbf{H}(z)$ has to be $\mathrm{RP}$ if $\mathbf{A}(z) \mathbf{H}(z)$ is $\mathrm{RP}$.

Conversely, let $\mathbf{x}(n)$ be the input of the cascaded system $\mathbf{H}(z) \mathbf{A}(z)$ and $\mathbf{y}(n)$ be the output of $\mathbf{H}(z) \mathbf{A}(z)$. In addition we assume $\mathbf{A}(z)=z^{-m} \mathbf{A}^{\prime}(z)$ where $m$ is a nonnegative integer and $\mathbf{A}^{\prime}(z)$ is a causal Type A RP system with first coefficient matrix nonzero. Then the cascaded system can be viewed as a cascade of $\mathbf{A}^{\prime}(z)$ followed by $\mathbf{H}(z) z^{-m}$. Let $\mathbf{x}_{1}(n)$ be the output of the subsystem $\mathbf{A}^{\prime}(z)$ and the input of the subsystem $\mathbf{H}(z) z^{-m}$. Suppose $\mathbf{H}(z)$ is not $\mathrm{RP}$, then $\mathbf{H}(z) z^{-m}$ is also not RP. So there exists input sequence $\mathbf{x}_{1}(n)$ such that the output $\mathbf{y}(n)$ of $\mathbf{H}(z) z^{-m}$ is not rich. By Lemma 3 we can find an input $\mathbf{x}(n)$ for $\mathbf{A}^{\prime}(z)$ such that the output is $\mathbf{x}_{1}(n)$, and by Lemma 1 this $\mathbf{x}(n)$ must be rich. So we can use this $\mathbf{x}(n)$ to be the input of the whole system $\mathbf{H}(z) \mathbf{A}(z)$ and generate the nonrich output $\mathbf{y}(n)$. So $\mathbf{H}(z)$ must be RP if $\mathbf{H}(z) \mathbf{A}(z)$ is RP.

Proof of Theorem 4: Let $\mathbf{B}(z)=\sum_{k=0}^{N} \mathbf{a}_{k} \mathbf{v}^{\dagger} z^{-k}$. Assume $\|\mathbf{v}\|=1$. Take an arbitrary nonzero row vector $\mathbf{w}^{\dagger}$ and let $\mathbf{H}_{1}(z)=\mathbf{v} \mathbf{w}^{\dagger}$, which is a singular constant matrix and is obviously non-RP. Then it can be shown that $\mathbf{B}(z) \mathbf{H}_{1}(z)=$ $\sum_{k=0}^{N} \mathbf{a}_{k} \mathbf{w}^{\dagger} z^{-k}$ is also RP!

Suppose $\mathbf{a}_{0}$ and $\mathbf{a}_{N}$ are nonzero. Let $\mathbf{H}_{2}(z)=\mathbf{I}_{M}+$ $\mathbf{a}_{0} \mathbf{a}_{N}^{\dagger} z^{-1}$. Since the coefficient matrices have different ranks, $\mathbf{H}_{2}(z)$ is not RP. But the product $\mathbf{H}_{2}(z) \mathbf{B}(z)$ can be shown to have the form $\sum_{k=0}^{N+1} \mathbf{b}_{k} \mathbf{v}^{\dagger} z^{-k}$, where $\mathbf{b}_{0}=\mathbf{a}_{0}, \mathbf{b}_{k}=\mathbf{a}_{k}+c_{k-1} \mathbf{a}_{0}$ for $1 \leq k \leq N$, and $\mathbf{b}_{N+1}=\mathbf{a}_{0} c_{N}$, where $c_{k}=\mathbf{a}_{k}^{\dagger} \mathbf{a}_{N}$ are constants. One can verify that the matrix $\left[\mathbf{b}_{0} \mathbf{b}_{1} \cdots \mathbf{b}_{N}\right]$ is a full rank matrix and hence $\mathbf{H}_{2}(z) \mathbf{B}(z)$ is Type B RP.

\section{E. Paraunitary and Unimodular Matrices}

In Examples 1 and 2, we have seen that PU matrices and unimodular matrices are not necessarily RP. Using Theorem 1, we can actually show that FIR PU and unimodular matrices cannot preserve richness unless they are constant matrices (with a possible delay in the PU case).

Corollary 3: If a PU matrix $\mathbf{H}(z)$ is $\mathrm{RP}$, then $\mathbf{H}(z)$ is a constant unitary matrix or a delayed version of it.

Proof: Without loss of generality, assume $\mathbf{h}(0) \neq \mathbf{0}$. Suppose $\mathbf{H}(z)=\sum_{k=0}^{N} \mathbf{h}(k) z^{-k}$ is PU and richness preserving but not a constant matrix (i.e., $N>0$ and $\mathbf{h}(N)$ is nonzero). From properties of PU matrices, we know both $\mathbf{h}(0)$ and $\mathbf{h}(N)$ are singular [4]. Using Corollary 2 of Theorem 1 , there exist row vector $\mathbf{v}^{\dagger}$ and $N+1$ column vectors $\mathbf{a}_{0}, \mathbf{a}_{1}, \cdots, \mathbf{a}_{N}$ such that $\mathbf{H}(z)=\sum_{k=0}^{N} \mathbf{a}_{k} \mathbf{v}^{\dagger} z^{-k}$. So $\widetilde{\mathbf{H}}(z)=\sum_{l=0}^{N} \mathbf{v a}_{l}^{\dagger} z^{l}$ and

$$
\widetilde{\mathbf{H}}(z) \mathbf{H}(z)=\sum_{k=0}^{N} \sum_{l=0}^{N} \mathbf{v a}_{l}^{\dagger} \mathbf{a}_{k} \mathbf{v}^{\dagger} z^{-(k-l)} .
$$

The constant term $\left(z^{0}\right)$ of $\widetilde{\mathbf{H}}(z) \mathbf{H}(z)$ would be

$$
\sum_{k=0}^{N} \mathbf{v} \mathbf{a}_{k}^{H} \mathbf{a}_{k} \mathbf{v}^{\dagger}=\left[\sum_{k=0}^{N} \mathbf{a}_{k}^{\dagger} \mathbf{a}_{k}\right] \mathbf{v} \mathbf{v}^{\dagger}
$$

since $\mathbf{a}_{k}^{\dagger} \mathbf{a}_{k}$ are all constants. The matrix $\mathbf{v v}^{\dagger}$ obviously has rank one. This contradicts $\widetilde{\mathbf{H}}(z) \mathbf{H}(z)=\mathbf{I}_{M}$, completing the proof. 
Corollary 4: If a unimodular matrix $\mathbf{H}(z)$ is richness preserving, then $\mathbf{H}(z)$ is a constant matrix.

Proof: If $\mathbf{H}(z)=\sum_{k=0}^{N} \mathbf{h}(k) z^{-k}$ is unimodular, $\operatorname{det}(\mathbf{h}(0))=\operatorname{det}(\mathbf{H}(\infty))=1$. So $\mathbf{h}(0)$ must be nonsingular. If $\mathbf{H}(z)$ is also RP, it must satisfy condition a) in Theorem 1. Then $\mathbf{H}(z)=\left(\sum_{k=0}^{N} g_{k} z^{-k}\right) \mathbf{A}$ and $\operatorname{det}(\mathbf{H}(z))=\left(\sum_{k=0}^{N} g_{k} z^{-k}\right)^{M} \operatorname{det}(\mathbf{A})=1$, where $\mathbf{A}$ is nonsingular. So we have $g_{k}=0$ for $k>0$ and hence $\mathbf{H}(z)$ must be a constant matrix.

\section{Strict Definition of Richness}

In practical applications, the new definition of richness given below might be more useful.

Definition 2: A sequence of $M \times 1$ vectors $\mathbf{x}(n), n \geq 0$ is said to be strictly rich (SR) if for any positive integer $n_{0}$, there exists an integer $K_{n_{0}}$ such that the matrix

$$
\left[\begin{array}{llll}
\mathbf{x}\left(n_{0}\right) & \mathbf{x}\left(n_{0}+1\right) & \cdots & \mathbf{x}\left(n_{0}+K_{n_{0}}\right)
\end{array}\right]
$$

has rank $M$.

Observe that a strictly rich signal is also rich according to the old definition. Conversely, a rich signal is not necessarily strictly rich. Furthermore, we will find that some systems that preserve richness according to the old definition no longer preserve strict richness. For example, we showed that Type A RP system $\mathbf{H}(z)=\left[\begin{array}{ll}1 & 0 \\ 0 & 1\end{array}\right]+z^{-1}\left[\begin{array}{ll}1 & 0 \\ 0 & 1\end{array}\right]$ preserves richness. However, if we let $\mathbf{x}(2 n)=\left[\begin{array}{ll}1 & 0\end{array}\right]^{T}$ and $\mathbf{x}(2 n+1)=\left[\begin{array}{ll}0 & 1\end{array}\right]^{T}$ for all nonnegative $n$, then the output would be $\mathbf{y}(0)=\left[\begin{array}{ll}1 & 0\end{array}\right]^{T}$ and $\mathbf{y}(n)=\left[\begin{array}{ll}1 & 1\end{array}\right]^{T}$ for any positive $n$. Here, the input $\mathbf{x}(n)$ is both rich and strictly rich. But the output $\mathbf{y}(n)$ is not strictly rich. The necessary and sufficient condition for LTI systems to preserve strict richness is summarized in the following theorem.

Theorem 5: An $N$ th order, $M \times M$ polynomial matrix

$$
\mathbf{H}(z)=\sum_{k=0}^{N} \mathbf{h}(k) z^{-k}
$$

is a strictly rich preserving (SRP) LTI system if and only if there exists nonnegative integer $n$ and an invertible $M \times M$ matrix $\mathbf{A}$ such that $\mathbf{H}(z)=z^{-n} \mathbf{A}$.

In view of this theorem, we find if a system is SRP, then it is also RP. We will prove Theorems 1 and 5 together in Section VI.

\section{Proof of the Main Theorems}

\section{A. Sketch of the Proof}

In this section, we will prove Theorems 1 and 5 step by step. We will first show that conditions described in Theorems 1 and 5 are sufficient (Section VI-B). Then we will present Lemma 5, which shows that necessary conditions for Theorem 1 are also necessary for Theorem 5 (Section VI-C). From Sections VI-D to VI-F, necessary conditions of Theorem 1 will be developed. In particular, a term coefficient rank will be defined for all RP systems to denote the ranks of all nonzero coefficient matrices since they will prove to be the same (Section VI-E). The coefficient rank will later on prove to be either unity or $M$. Finally, for the case of unity coefficient rank, we will show condition b) is necessary, and for the case of full coefficient rank, condition a) is necessary (Section VI-F).
In Section VI-G, we will show that Type A and Type B RP systems cannot preserve strict richness unless it is a constant invertible matrix with a possible delay.

\section{B. Proof of Sufficiency}

We first prove conditions a) and b) in Theorem 1 are sufficient for preserving richness.

Proof: If $\mathbf{H}(z)$ satisfies condition a) by Theorem 1 in [2], it is RP. Suppose $\mathbf{H}(z)$ satisfies condition b) but is not RP. Then there exists a rich input $\mathbf{x}(n)$ such that the output $\mathbf{y}(n)$ is not rich, i.e., there exists a row vector $\mathbf{w}^{\dagger}$ such that $\mathbf{w}^{\dagger} \mathbf{y}(n)=$ $0, \forall n$. Using $\mathbf{y}(n)=\sum_{k=0}^{N} \mathbf{h}(k) \mathbf{x}(n-k)$, we have the following equations:

$$
\begin{aligned}
\left(\mathbf{w}^{\dagger} \mathbf{a}_{0}\right)\left(\mathbf{v}^{\dagger} \mathbf{x}(0)\right) & =0 \\
\left(\mathbf{w}^{\dagger} \mathbf{a}_{0}\right)\left(\mathbf{v}^{\dagger} \mathbf{x}(1)\right)+\left(\mathbf{w}^{\dagger} \mathbf{a}_{1}\right)\left(\mathbf{v}^{\dagger} \mathbf{x}(0)\right) & =0 \\
& \vdots \\
\sum_{k=0}^{N}\left(\mathbf{w}^{\dagger} \mathbf{a}_{k}\right)\left(\mathbf{v}^{\dagger} \mathbf{x}(N-k)\right) & =0 .
\end{aligned}
$$

If $\mathbf{v}^{\dagger} \mathbf{x}(0)$ is not zero, then from the first equation, we have $\mathbf{w}^{\dagger} \mathbf{a}_{0}=0$. Substituting this into the second equation, we get

$$
\left(\mathbf{w}^{\dagger} \mathbf{a}_{1}\right)\left(\mathbf{v}^{\dagger} \mathbf{x}(0)\right)=0 .
$$

So $\mathbf{w}^{\dagger} \mathbf{a}_{1}$ has to be zero. Repeating these substitutions, we will have $\mathbf{w}^{\dagger} \mathbf{a}_{k}=0, \forall k, 0 \leq k \leq N$. This contradicts the statement that $\left[\mathbf{a}_{0}, \mathbf{a}_{1}, \ldots, \mathbf{a}_{N}\right]$ has rank $M$. So $\mathbf{v}^{\dagger} \mathbf{x}(0)$ has to be zero. Substituting this into (1) and repeating the same derivations, we will have $\mathbf{v}^{\dagger} \mathbf{x}(1)=\mathbf{0}$ as well. Repeating this, we get $\mathbf{v}^{\dagger} \mathbf{x}(n)=$ 0 for all $n$. This violates richness of the input $\mathbf{x}(n)$. So condition b) is also sufficient.

The sufficiency for Theorem 5 is self-evident.

\section{Relationship Between RP and SRP Systems}

We know that strict richness implies richness but not vice versa. So, it is not obvious that an SRP system is also RP. We will show, however, that this is the case.

Lemma 5: Given $M \times M$ polynomial matrix $\mathbf{H}(z)=$ $\sum_{k=0}^{N} \mathbf{h}(k) z^{-k}$, if there exists a rich causal signal $\mathbf{x}(n)$ which has a finite support such that $\mathbf{y}(n)=\sum_{k=0}^{N} \mathbf{h}(k) \mathbf{x}(n-k)$ is nonrich, then $\mathbf{H}(z)$ is neither RP nor SRP.

Proof: By definition, $\mathbf{H}(z)$ is not RP. Suppose $\mathbf{x}(n)$ has length $L$, that is, $\mathbf{x}(n)=\mathbf{0}$ for all $n \geq L$. Then it is clear that $\mathbf{y}(n)=\sum_{k=0}^{N} \mathbf{h}(k) \mathbf{x}(n-k)=\mathbf{0}$ for all $n \geq L+N$. Now consider a new signal $\mathbf{x}^{\prime}(n)=\mathbf{x}(n \bmod (N+L))$. Since $\mathbf{x}(n)$ is rich, we have $\mathbf{x}^{\prime}(n)$ is strictly rich. Then using the facts that $\mathbf{x}(n)=\mathbf{0}$ for all $n \geq L$ and that $\mathbf{y}(n)$ is not rich, we find $\mathbf{y}^{\prime}(n)=\sum_{k=0}^{N} \mathbf{h}(k) \mathbf{x}^{\prime}(n-k)=\sum_{k=0}^{N} \mathbf{h}(k) \mathbf{x}((n-$ $k) \bmod (N+L))=\sum_{k=0}^{N} \mathbf{h}(k) \mathbf{x}([n \bmod (N+L)]-k)=$ $\mathbf{y}(n \bmod (N+L))$ is also not strictly rich. Therefore, $\mathbf{H}(z)$ is not SRP.

We will use this lemma to show that SRP is stronger than RP. In the following lemmas, we will derive necessary conditions for Theorem 1 by constructing rich input signals that have nonrich output for a system that does not satisfy these conditions. All of the input signals we construct will have finite support, and hence the necessary conditions for Theorem 1 are also those for Theorem 5. 


\section{Lemmas for Proof of Necessity}

Lemma 6: If an $M \times M$ polynomial matrix $\mathbf{H}(z)=$ $\sum_{k=0}^{N} \mathbf{h}(k) z^{-k}$ is $\mathrm{RP}$, then there exist $M \times M$ diagonal matrices $\mathbf{D}_{k}$ and an $M \times M$ constant matrix $\mathbf{A}$, each row of which is nonzero such that $\mathbf{h}(k)=\mathbf{D}_{k} \mathbf{A}$.

Proof: For $0 \leq k \leq N$, we assume

$$
\mathbf{h}(k)=\left[\begin{array}{llll}
\mathbf{a}_{1 k} & \mathbf{a}_{2 k} & \cdots & \mathbf{a}_{M k}
\end{array}\right]^{T}
$$

where

$$
\mathbf{a}_{i k}^{T}=\left[\begin{array}{llll}
a_{i 1 k} & a_{i 2 k} & \cdots & a_{i M k}
\end{array}\right]
$$

is the $i$ th row of $\mathbf{h}(k)$. Focusing on the $i$ th row of $\mathbf{H}(z)$, we use

$$
\mathbf{b}_{k}^{T}=\left[\begin{array}{llll}
b_{1 k} & b_{2 k} & \cdots & b_{M k}
\end{array}\right]
$$

to denote $\mathbf{a}_{i k}^{T}$ for simplicity. Since $\mathbf{H}(z)$ is richness preserving, any row of $\mathbf{H}(z)$ cannot be all zeros. So there exists $b_{j k}$ that is nonzero. Without loss of generality, assume $b_{10} \neq 0$. Construct the input as

$$
\begin{gathered}
\mathbf{x}(0)=b_{20} \mathbf{e}_{1}-b_{10} \mathbf{e}_{2} \\
\mathbf{x}(1)=b_{21} \mathbf{e}_{1}-b_{11} \mathbf{e}_{2} \\
\vdots \\
\mathbf{x}(N)=b_{2 N} \mathbf{e}_{1}-b_{1 N} \mathbf{e}_{2} \\
\mathbf{x}(m(N+1)+k)=b_{(m+2), k} \mathbf{e}_{1}-b_{1 k} \mathbf{e}_{m+2}, \\
\quad 0 \leq m \leq M-2, \quad 0 \leq k \leq N .
\end{gathered}
$$

For simplicity, we will use $\mathbf{x}_{m}(k)$ to denote $\mathbf{x}(m(N+1)+k)$.

By the definitions above, one can verify the following things for $0 \leq m \leq M-2,0 \leq k, l \leq N$ :

1) $\mathbf{b}_{k}^{T} \mathbf{x}_{m}(k)=0$

2) $\mathbf{b}_{k}^{T} \mathbf{x}_{m}(l)+\mathbf{b}_{l}^{T} \mathbf{x}_{m}(k)=0$.

Using these results, it can be shown that

$$
\begin{aligned}
{[\mathbf{y}(n)]_{i} } & =\left[\sum_{k=0}^{N} \mathbf{h}(k) \mathbf{x}(n-k)\right]_{i} \\
& =\sum_{k=0}^{N} \mathbf{b}_{k}^{T} \mathbf{x}(n-k)=0 .
\end{aligned}
$$

Hence the output $\mathbf{y}(n)$ is not rich. Since $\mathbf{H}(z)$ is richness preserving, $\mathbf{x}(n)$ must also be not rich. Define the $M \times M$ matrix

$$
\mathbf{X}_{1}=\left[\begin{array}{llllll}
\mathbf{x}(0) & \mathbf{x}(1) & \mathbf{x}_{1}(0) & \mathbf{x}_{2}(0) & \cdots & \mathbf{x}_{M-2}(0)
\end{array}\right] .
$$

One can verify the absolute value of the determinant of $\mathbf{X}_{1}$ is $\left|\operatorname{det}\left(\mathbf{X}_{1}\right)\right|=\left|b_{10}\right|^{M-2}\left|b_{10} b_{21}-b_{11} b_{20}\right|$. Since $\mathbf{x}(n)$ is not rich, $\operatorname{det}\left(\mathbf{X}_{1}\right)=0$. Since $b_{10}$ is nonzero, we get $b_{10} b_{21}=b_{11} b_{20}$, or $b_{21}=d_{i 1} b_{20}$, where $d_{i 1}$ is chosen as $b_{11} / b_{10}$. Now we define another $M \times M$ matrix by replacing $\mathbf{x}(1)$ in the definition of $\mathbf{X}_{1}$ with another $\mathbf{x}_{m}(1)$, and we obtain $b_{(m+2), 1}=d_{i 1} b_{(m+2), 0}$. These results for all $m$ imply that $\mathbf{b}_{1}=d_{i 1} \mathbf{b}_{0}$, or $\mathbf{a}_{i 1}=d_{i 1} \mathbf{a}_{i 0}$.

If we replace $\mathbf{x}(1)$ in the definition of $\mathbf{X}_{1}$ with $\mathbf{x}_{m}(k)$, we can show that $\exists d_{i k}$ such that $\mathbf{b}_{k}=d_{i k} \mathbf{b}_{0}$, or $\mathbf{a}_{i k}=d_{i k} \mathbf{a}_{i 0}$. Finally, define $\mathbf{v}_{i}=\mathbf{a}_{i 0}$ and $d_{i 0}=1$, then we have $\mathbf{a}_{i k}=d_{i k} \mathbf{v}_{i}$ for all $i$ and $k$. The reader has to note that here we assign $\mathbf{v}_{i}$ as $\mathbf{a}_{i 0}$ just because of the assumption that $b_{10}$ is nonzero without loss of generality. If $b_{10}=0$, we can find another $b_{j k}$ that is nonzero and do similar derivation, and $\mathbf{v}_{i}$ here will be assigned as another $\mathbf{a}_{i k}$ rather than $\mathbf{a}_{i 0}$. After all, $\exists d_{i k}, \mathbf{v}_{i} \neq \mathbf{0}$ such that $\mathbf{a}_{i k}=d_{i k} \mathbf{v}_{i}$ is still true for all $i$ and $k$. Now we simply assign

$$
\mathbf{A}=\left[\begin{array}{llll}
\mathbf{v}_{1} & \mathbf{v}_{2} & \cdots & \mathbf{v}_{M}
\end{array}\right]^{T}
$$

and

$$
\mathbf{D}_{k}=\operatorname{diag}\left[\begin{array}{llll}
d_{1 k} & d_{2 k} & \cdots & d_{M k}
\end{array}\right]
$$

Then the proof is complete.

Lemma 6 will play an important role in the proof of necessity for both conditions a) and b) of Theorem 1. Some other useful lemmas will be presented here.

Lemma 7: $\mathbf{H}(z)$ is $\mathrm{RP}$ if and only if $\mathbf{A H}(z)$ is RP, where $\mathbf{A}$ is any nonsingular $M \times M$ matrix.

Proof: This lemma becomes obvious when we recognize that $\mathbf{x}(n)$ is rich if and only if $\mathbf{A x}(n)$ is rich for any nonsingular matrix A.

Lemma 8: $\mathbf{H}(z)$ is RP if and only if $z^{-k} \mathbf{H}(z)$ is RP, where $k$ is any nonnegative integer.

Proof: This is self-evident.

Lemma 7 allows us to do invertible row operations on $\mathbf{H}(z)$ since each invertible row operation corresponds to a nonsingular matrix. Lemma 8 allows us to assume $\mathbf{h}(0) \neq \mathbf{0}$ for an RP matrix $\mathbf{H}(z)$.

\section{E. Coefficient Rank of an RP System}

Lemma 9: For an FIR system $\mathbf{H}(z)=\sum_{k=0}^{N} \mathbf{h}(k) z^{-k}$ that preserves richness, the ranks of all nonzero coefficient matrices must be the same. We call this value the coefficient rank of an RP system.

Proof: Suppose $\mathbf{h}(j)$ has the smallest rank $\rho$ among all nonzero $\mathbf{h}(k)(\rho>0)$. By Lemma 7, we can do invertible row operations on $\mathbf{H}(z)$ such that $\mathbf{h}(j)$ can be expressed as

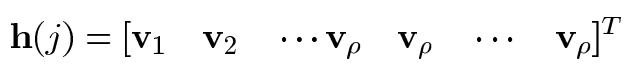

where $\mathbf{v}_{1}, \ldots, \mathbf{v}_{\rho}$ are linearly independent nonzero column vectors. By Lemma 6, there exist a constant matrix $\mathbf{A}$ and a diagonal matrix $\mathbf{D}_{j}$ such that $\mathbf{h}(j)=\mathbf{D}_{j} \mathbf{A}$. Since each row of $\mathbf{h}(j)$ is nonzero, all diagonal entries of $\mathbf{D}_{j}$ must be nonzero and $\mathbf{A}$ also has rank $\rho$.

Now for any other nonzero coefficient matrix $\mathbf{h}(k)$, there exists a diagonal matrix $\mathbf{D}_{k}$ such that $\mathbf{h}(k)=\mathbf{D}_{k} \mathbf{A}$. So $\operatorname{rank}(\mathbf{h}(k)) \leq \operatorname{rank}(\mathbf{A})=\rho$. Since $\mathbf{h}(j)$ has the smallest nonzero $\operatorname{rank} \rho$, we have $\operatorname{rank}(\mathbf{h}(k))=\rho$.

In the following two lemmas, we will prove the coefficient rank of an RP system can only be unity or $M$.

Lemma 10: If an RP system with the form $\mathbf{H}(z)=$ $\sum_{k=0}^{N} \mathbf{D}_{k} \mathbf{A} z^{-k}$ has coefficient rank $\rho$, where $\mathbf{D}_{k}$ 's are diagonal matrices and $\mathbf{A}$ is a constant matrix, then $\operatorname{rank}(\mathbf{A})=\rho$.

Proof: If $\mathbf{H}(z)$ has only one nonzero coefficient matrix, then the statement is self-evident. Now we assume $\mathbf{H}(z)$ has at least two nonzero coefficient matrices and without loss of generality, assume $\mathbf{h}(0)$ and $\mathbf{h}(l)$ are nonzero. Since $\mathbf{h}(0)=\mathbf{D}_{0} \mathbf{A}$, we have $\operatorname{rank}(\mathbf{A}) \geq \operatorname{rank}(\mathbf{h}(0))=\rho$. Suppose $\operatorname{rank}(\mathbf{A})>\rho$, then without loss of generality we can assume the first $\rho+1$ rows of $\mathbf{A}$, namely $\mathbf{a}_{1}^{T}, \ldots, \mathbf{a}_{\rho}^{T}, \mathbf{a}_{\rho+1}^{T}$, are linearly independent. 
Since $\operatorname{rank}(\mathbf{h}(0))=\rho$, we can further assume the first row of $\mathbf{h}(0)$ is zero while rows $2,3, \ldots,(\rho+1)$ are nonzero. Let $d_{i k}$ denote the $i$ th diagonal entry of $\mathbf{D}_{k}$. Now we have $d_{10}=0$ and $d_{i 0} \neq 0$ for $2 \leq i \leq \rho+1$. Since $\mathbf{H}(z)$ is $\mathrm{RP}$, there exists a coefficient matrix whose first row is nonzero. Assume $\mathbf{h}(l)$ satisfies this and thus $d_{1 l} \neq 0$. Since $\operatorname{rank}(\mathbf{h}(l))=\rho$, at least one of the first $\rho+1$ rows of $\mathbf{h}(l)$ must be zero. Assume the second row is zero which means $d_{2 l}=0$. By Lemma 7 we can do an invertible row operation on $\mathbf{H}(z)$ by adding the second row into the first row and produce another RP system $\mathbf{H}^{\prime}(z)=\sum_{k=0}^{N} \mathbf{h}^{\prime}(k) z^{-k}$. Now the first rows of $\mathbf{h}^{\prime}(0)$ and $\mathbf{h}^{\prime}(l)$ are $d_{20} \mathbf{a}_{2}^{T}$ and $d_{1 l} \mathbf{a}_{1}^{T}$, respectively. They are both nonzero and are linearly independent. This makes it impossible for $\mathbf{H}^{\prime}(z)$ to be written as the form in Lemma 6 and causes a contradiction. So $\operatorname{rank}(\mathbf{A})=\rho$ must be true.

Lemma 11: The coefficient rank of an RP system can only be unity or $M$.

Proof: Suppose there exists an RP matrix $\mathbf{H}(z)$ that has a coefficient rank $\rho$ where $2 \leq \rho \leq M-1$. By Lemmas 2 and 3 , we can assume $\mathbf{h}(0) \neq 0$ and do invertible row operations on $\mathbf{H}(z)$ such that

$$
\mathbf{h}(0)=\left[\begin{array}{lllllll}
\mathbf{a}_{1} & \mathbf{a}_{2} & \cdots & \mathbf{a}_{\rho} & \mathbf{0} & \cdots & \mathbf{0}
\end{array}\right]^{T} .
$$

Since $\rho<M$, the last row of $\mathbf{h}(0)$ must be a zero vector. The last rows of other $\mathbf{h}(k)$, however, cannot be all zeros. By Lemma 6 , there exist a constant matrix $\mathbf{A}$ and a diagonal matrix $\mathbf{D}_{0}$ such that $\mathbf{h}(0)=\mathbf{D}_{0} \mathbf{A}$. By Lemma 10 , we know $\operatorname{rank}(\mathbf{A})=\rho$. So the last row of $\mathbf{A}$, namely $\mathbf{v}_{2}^{T}$, must be a linear combination of $\mathbf{a}_{1}^{T}, \mathbf{a}_{2}^{T}, \cdots, \mathbf{a}_{\rho}^{T}$.

Since $\rho \geq 2$, we can find an $i, 1 \leq i \leq \rho$ such that $\mathbf{a}_{i}$ and $\mathbf{v}_{2}$ are linearly independent. For convenience, we define $\mathbf{v}_{1}=\mathbf{a}_{i}$. Now we can find a set of linearly independent vectors $\mathbf{w}_{1}, \mathbf{w}_{2}, \ldots, \mathbf{w}_{M}$ such that $\mathbf{w}_{1}$ is orthogonal to $\mathbf{v}_{2}, \mathbf{w}_{2}$ is orthogonal to $\mathbf{v}_{1}$, and $\mathbf{w}_{3}, \mathbf{w}_{4}, \ldots, \mathbf{w}_{M}$ are orthogonal to both $\mathbf{v}_{1}$ and $\mathbf{v}_{2}$. (For example, we can let $\mathbf{w}_{1}=\mathbf{v}_{1}-\left(\mathbf{v}_{1}^{T} \mathbf{v}_{2} /\left\|\mathbf{v}_{2}\right\|^{2}\right) \mathbf{v}_{2}$.) Furthermore, we can assume $\mathbf{v}_{1}^{T} \mathbf{w}_{1}=\mathbf{v}_{2}^{T} \mathbf{w}_{2}=1$.

Now we focus on the $i$ th and the $M$ th rows of $\mathbf{H}(z)$. They are $\mathbf{v}_{1}^{T}+\sum_{n=1}^{N} p_{n} \mathbf{v}_{1}^{T} z^{-n}$ and $\sum_{n=1}^{N} q_{n} \mathbf{v}_{2}^{T} z^{-n}$, respectively, where $\left\{q_{n}\right\}_{n=1}^{N}$ are not all zeros. Construct the input sequence as

$$
\begin{aligned}
\mathbf{x}(n) & =\mathbf{w}_{n+3}, \quad 0 \leq n \leq M-3 \\
\mathbf{x}(M-2) & =\mathbf{w}_{2} \\
\mathbf{x}(M-2+k) & =q_{k} \mathbf{w}_{1}+p_{k} \mathbf{w}_{2}, \quad 1 \leq k \leq N \\
\mathbf{x}(n) & =\mathbf{0}, \quad \forall n \geq M+N-1 .
\end{aligned}
$$

Then one can verify that $[\mathbf{y}(n)]_{i}=[\mathbf{y}(n)]_{M}$ for all $n$ and hence $\mathbf{y}(n)$ is not rich. However, the input $\mathbf{x}(n)$ is rich. This contradicts the assumption that $\mathbf{H}(z)$ is RP. So the coefficient rank of $\mathbf{H}(z)$ can only be unity or $M$.

\section{F. Completion of Proof of Necessity for RP Systems}

Now we are ready to prove conditions a) and b) are necessary for the richness-preserving property.

Proof: Let $\mathbf{H}(z)$ be RP. By Lemma 8, we assume $\mathbf{h}(0) \neq$ 0. If $\mathbf{h}(0)$ is singular, the coefficient rank of $\mathbf{H}(z)$ must be unity by Lemma 11 . So there exist a nonzero row vector $\mathbf{v}^{T}$ and column vectors $\mathbf{a}_{0}, \mathbf{a}_{1}, \cdots, \mathbf{a}_{N}$ such that $\mathbf{h}(k)=\mathbf{a}_{k} \mathbf{v}^{T}$.
Now we only need to prove $\left[\mathbf{a}_{0}, \mathbf{a}_{1}, \ldots, \mathbf{a}_{N}\right]$ has full rank. If this is not true, we can find an annihilator $\mathbf{w}^{T}$ for all $\mathbf{a}_{k}$. Then no matter what the input is, the output $\mathbf{y}(n)$ will have an annihilator $\mathbf{w}^{T}$, and thus $\mathbf{H}(z)$ becomes richness destroying. So $\left[\mathbf{a}_{0}, \mathbf{a}_{1}, \ldots, \mathbf{a}_{N}\right]$ must have rank $M$, and thus condition $\left.\mathbf{b}\right)$ must be true.

If $\mathbf{h}(0)$ is nonsingular, the coefficient rank of $\mathbf{H}(z)$ must be $M$. By Lemma 7 we assume $\mathbf{h}(0)=\mathbf{I}$ without loss of generality. Using Lemma 6, $\mathbf{H}(z)$ must have the form

$$
\mathbf{H}(z)=\mathbf{I}+\sum_{k=1}^{N} z^{-k} \operatorname{diag}\left(\left[\begin{array}{llll}
a_{k 1} & a_{k 2} & \cdots & a_{k M}
\end{array}\right]\right) .
$$

Suppose there exist $i, j, k$ such that $a_{k i} \neq a_{k j}$ and $i \neq j$. Let

$$
\begin{aligned}
\mathbf{x}(0) & =\mathbf{e}_{i}+\mathbf{e}_{j} \\
\mathbf{x}(n) & =a_{n j} \mathbf{e}_{i}+a_{n i} \mathbf{e}_{j}, \quad 1 \leq n \leq N \\
\{\mathbf{x}(n)\}_{n=N+1}^{M+N-2} & =\left\{\mathbf{e}_{l} \mid 1 \leq l \leq M, l \neq i, l \neq j\right\} \\
\mathbf{x}(n) & =\mathbf{0}, \quad \forall n \geq M+N-1 .
\end{aligned}
$$

Since $a_{k i} \neq a_{k j}$, one can verify that $\mathbf{x}(n)$ is rich. It is also easy to verify the following for $1 \leq k \leq N$ :

1) $[\mathbf{x}(0)]_{i}=[\mathrm{x}(0)]_{j}$;

2) $a_{k i}\left[\mathbf{x}_{k}\right]_{i}=a_{k j}[\mathbf{x}(k)]_{j}$;

3) $[\mathbf{x}(k)]_{i}+a_{k i}[\mathbf{x}(0)]_{i}=[\mathbf{x}(k)]_{j}+a_{k j}[\mathbf{x}(0)]_{j}$;

4) $a_{l i}[\mathbf{x}(k)]_{i}+a_{k i}[\mathbf{x}(l)]_{i}=a_{l j}[\mathbf{x}(k)]_{j}+a_{k j}[\mathbf{x}(l)]_{j}, 1 \leq l \leq$ $N$

5) $a_{k i}[\mathbf{x}(l)]_{i}=a_{k j}[\mathbf{x}(l)]_{j}, N+1 \leq l \leq M+N-2$.

Using these facts, we can show $[\mathbf{y}(n)]_{i}=[\mathbf{y}(n)]_{j}, n \geq 0$, and hence $\mathbf{y}(n)$ is not rich. So in order to let $\mathbf{H}(z)$ preserve richness, $a_{k i}=a_{k j}$ must be true for any $i \neq j$ and any $k$. This means each coefficient matrix of $\mathbf{H}(z)$ is proportional to identity matrix and hence condition a) must be true.

The Proof of Theorem 1 is now complete. In addition, by Lemma 5, we know the necessary conditions in Theorem 1 are also necessary for SRP systems. In the next subsection, we will show that SRP systems require even stronger necessary conditions and complete the Proof of Theorem 5.

\section{G. Necessary Conditions for Preserving Strict Richness}

Lemma 12: If a Type A RP matrix $\mathbf{A}(z)$ preserves strict richness, then it must be a constant invertible matrix with a possible delay.

Proof: Assume $\mathbf{A}(z)=g(z) \mathbf{A}$, where $\mathbf{A}$ is an invertible constant matrix and $g(z)$ is a nonzero polynomial of $z^{-1}$. Suppose the contrary, then the polynomial $g(z)$ must have at least two terms and hence have at least one zero other than infinity. Suppose $g(\alpha)=0$. Let the input $\mathbf{x}(n)=\alpha^{n} \mathbf{A}^{-1} \mathbf{e}_{(n \bmod M)+1}$. Then for all $n \geq N$, we have

$$
\begin{aligned}
\sum_{i=1}^{M}[\mathbf{y}(n)]_{i} & =\sum_{i=1}^{M} \mathbf{e}_{i}^{T}\left[\sum_{k=0}^{N} g_{k} \mathbf{A} \mathbf{x}(n-k)\right] \\
& =\sum_{k=0}^{N} g_{k} \alpha^{n-k}\left[\sum_{i=1}^{M} \mathbf{e}_{i}^{T} \mathbf{e}_{((n-k) \bmod M)+1}\right] \\
& =\alpha^{n} g(\alpha)=0 .
\end{aligned}
$$

This means row vector $\left[\begin{array}{llll}1 & 1 & \cdots & 1\end{array}\right]$ is an annihilator of $\mathbf{y}(n)$ for all $n \geq N$. Therefore, $\mathbf{y}(n)$ is not strictly rich, and the proof is complete. 
Lemma 13: Type-B RP matrices $\mathbf{B}(z)$ do not preserve strict richness.

Proof: Suppose $\mathbf{B}(z)=\left(\sum_{k=0}^{N} \mathbf{a}_{k} z^{-k}\right) \mathbf{v}_{1}^{T}$ and assume $\left\|\mathbf{v}_{1}\right\|=1$. We can find $\mathbf{v}_{2}, \mathbf{v}_{3}, \ldots \mathbf{v}_{M}$ such that $\left\|\mathbf{v}_{k}\right\|=1$ and $\mathbf{v}_{i}^{T} \mathbf{v}_{j}=0, \forall i \neq j$. Let $\mathbf{w}_{1}=\mathbf{v}_{1}$ and $\mathbf{w}_{k}=\mathbf{v}_{1}+\mathbf{v}_{k}$ for $k \geq 2$. Then we have $\mathbf{v}_{1}^{T} \mathbf{w}_{k}=1, \forall k$. Let the input $\mathbf{x}(n)=$ $\mathbf{w}_{(n \bmod M)+1}$, and clearly it is strictly rich. However, for all $n \geq N$, we have $\mathbf{y}(n)=\sum_{k=0}^{N} \mathbf{a}_{k} \mathbf{v}_{1}^{T} \mathbf{x}(n-k)=\sum_{k=0}^{N} \mathbf{a}_{k}$, independent from $n$. This implies $\mathbf{y}(n)$ is not strictly rich. So Type B RP matrices cannot preserve strict richness.

Using Lemmas 5, 12, and 13, the Proof of Theorem 5 is now complete.

\section{RELATIONSHIP With PERsistent EXCITATION}

The definition of strict richness given in Section V happens to be related to the concept of "persistent excitation" in the literature on control theory. The property of persistent excitation is relevant to the stability and convergence of adaptive systems [11], [12]. The exact definition of persistent excitation can vary with respect to different applications. In [10, p. 1060], a sequence of $M \times 1$ vectors $\mathbf{x}(n)$ is called persistently exciting (PE) if there exists a finite integer $K$ such that the matrix

$$
\left[\begin{array}{llll}
\mathbf{x}(n) & \mathbf{x}(n+1) & \cdots & \mathbf{x}(n+K)
\end{array}\right]
$$

has rank $M$ for sufficiently large $n$. It is clear from the definition that PE implies SR. However, the converse is not true. This can be seen by constructing a sequence of $2 \times 1$ vectors $\mathbf{x}(n)$ as

$$
\mathbf{x}(n)= \begin{cases}{\left[\begin{array}{l}
1 \\
0
\end{array}\right],} & \text { if } n=2^{2 k} \quad \forall k \in \mathbb{N} \\
{\left[\begin{array}{l}
0 \\
1
\end{array}\right],} & \text { if } n=2^{2 k+1} \quad \forall k \in \mathbb{N} \\
{\left[\begin{array}{l}
0 \\
0
\end{array}\right],} & \text { otherwise. }\end{cases}
$$

It is readily verified that $\mathbf{x}(n)$ is SR but not PE. Although the definitions of SR and PE are not exactly equivalent, it can still be shown that for an LTI $M \times M$ system to preserve the property of PE is the same as to preserve SR, as stated in Theorem 5. The proof of this is rather involved and will be presented elsewhere.

An even stronger definition of PE can be found in [9] and [11]. Therein, the sequence $\mathbf{x}(n)$ is called persistently exciting if there exist positive integers $L, n_{0}$ and $\alpha>0$ such that for any vector $\mathbf{v} \in \mathbb{C}^{M}$ and any integer $n \geq n_{0},\left|\mathbf{v}^{\dagger} \mathbf{x}(k)\right|>\alpha$ for some $k$ satisfying $n \leq k \leq n+L$.

However, in many applications of control theory, the property of PE is applied to signals that are often called "regressors" [9], that is, the sequence of $M \times 1$ vectors $\mathbf{x}(n)$ comes from a sequence of scalars $x_{n}, n \geq 0$ and can be written as

$$
\mathbf{x}(n)=\left[\begin{array}{llll}
x_{n} & x_{n-1} & \cdots & x_{n-M+1}
\end{array}\right]^{T} .
$$

This constraint limits the degrees of freedom of choices of sequence $\mathbf{x}(n)$. If we take into account this constraint when studying PE signals, the problem of preserving PE becomes a totally different problem. The similarity between the definitions of SR and PE, nevertheless, suggests that there might exist some application in control or adaptive filtering to which the theory of richness preservation can be applied.

\section{CONCLUdING REMARKS AND OPEN ISSUES}

Necessary and sufficient conditions have been found for multiple-input multiple-output LTI FIR systems that are richness preserving and strict-richness preserving. The results show that most standard systems with memory do not generally preserve richness, including paraunitary and unimodular matrices. The similarity of, and relationship between, signal richness and persistent excitation has also been described and discussed. This relation suggests that there might be some applications of the results of this paper in the control theory literature.

Under the definitions of richness considered in this paper, it remains to investigate conditions on infinite-impulse-response (IIR) systems that preserve richness. It is also interesting to consider the case where the input and the output of the LTI system have different sizes.

Another issue of interest is the evaluation of the probability for an LTI system to preserve richness. For an LTI system that does not satisfy necessary conditions in Theorem 1, we can manage to find a rich input sequence such that the output of the system is not rich. In practical applications, however, the probability of appearance of such input could almost be zero! This suggests there may exist some LTI systems that, although not satisfying necessary conditions of Theorems 1 and 5, still preserve richness with probability one. These systems would still be very useful in practical applications. The RP conditions for such systems are characterized probabilistically and furthermore depend on the statistics of the class of allowed inputs. A study of such systems could be challenging and important.

It would also be of interest to study the case of wide-sensestationary (WSS) signals. In this case, richness can be defined with respect to the autocorrelation matrices (e.g., nonsingularity) of the signal. Development of RP conditions is equivalent to finding the conditions under which an LTI system preserves such nonsingular property.

\section{REFERENCES}

[1] R. A. Horn and C. R. Johnson, Matrix Analysis. Cambridge, U.K.: Cambridge Univ. Press, 1985.

[2] P. P. Vaidyanathan and B. Su, "Staying rich: LTI systems which preserve signal richness," in Proc. IEEE Int. Conf. Acoustics, Speech, Signal Processing, vol. 4, Philadelphia, PA, Mar. 2005, pp. 249-252.

[3] B. Su and P. P. Vaidyanathan, "Necessary and sufficient conditions for LTI systems to preserve signal richness," in Proc. IEEE Int. Symp. Circuits Systems, vol. 4, Kobe, Japan, May 2005, pp. 3035-3038.

[4] P. P. Vaidyanathan, Multirate Systems and Filter Banks. Englewood Cliffs, NJ: Prentice-Hall, 1993.

[5] A. Scaglione, G. B. Giannakis, and S. Barbarossa, "Redundant filter bank precoders and equalizers Part II: Synchronization and direct equalization," IEEE Trans. Signal Process., vol. 47, no. 7, pp. 2007-2002, Jul. 1999.

[6] S.-M. Phoong and K.-Y. Chang, "Antipodal paraunitary matrices and their application to OFDM systems," IEEE Trans. Signal Process., vol. 53, no. 4, pp. 1374-1386, Apr. 2005.

[7] P. P. Vaidyanathan and B. Vrcelj, "Transmultiplexers as precoders in modern digital communication: A tutorial review," in IEEE Int. Symp. Circuits Systems, vol. 5, Vancouver, BC, Canada, May 2004, pp. 405-412.

[8] P. P. Vaidyanathan and B. Su, "Remarks on certain new methods for blind identification of FIR channels," in Proc. IEEE Asilomar Conf. Signals, Systems, Computers, vol. 1, Pacific Grove, CA, Nov. 2004, pp. 832-836. 
[9] S. Haykin, Adaptive Filter Theory, 4th ed. Upper Saddle River, NJ: Prentice-Hall, 2002.

[10] A. H. Sayed, Fundamentals of Adaptive Filtering. New York: Wiley, 2003.

[11] W. A. Sethares et al., "Parameter drift in LMS adaptive filters," IEEE Trans. Acoust., Speech, Signal Process., vol. ASSP-34, no. 4, pp. 868-879, Aug. 1986.

[12] R. R. Bitmead, "Persistence of excitation conditions and the convergence of adaptive schemes," IEEE Trans. Inf. Theory, vol. IT-30, no. 2, pp. 183-191, Mar. 1986.

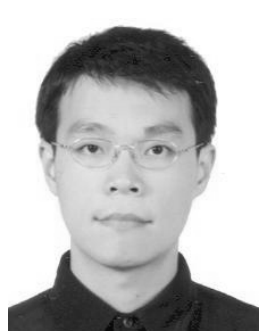

Borching Su ( $\left.\mathrm{S}^{\prime} 00\right)$ was born in Tainan, Taiwan, R.O.C., on October 8, 1978. He received the B.S. and M.S. degrees in electrical engineering and communication engineering, both from National Taiwan University (NTU), Taipei, Taiwan, R.O.C., in 1999 and 2001, respectively. He is currently working toward the Ph.D. degree in the field of digital signal processing at the California Institute of Technology (Caltech), Pasadena. His current research interests include multirate systems and their applications on digital communications

Mr. Su was awarded the Moore Fellowship from Caltech in 2003.

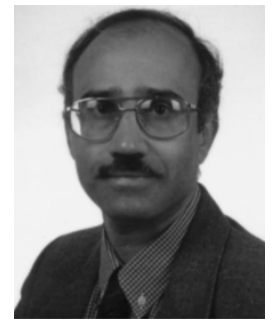

P. P. Vaidyanathan (S'80-M'83-SM'88-F'91) was born in Calcutta, India, on October 16, 1954. He received the B.Sc. (Hons.) degree in physics and the B.Tech. and M.Tech. degrees in radiophysics and electronics from the University of Calcutta, India, in 1974, 1977, and 1979, respectively, and the Ph.D. degree in electrical and computer engineering from the University of California, Santa Barbara, in 1982.

From September 1982 to March 1983, he was a Postdoctoral Fellow at the University of California, Santa Barbara. In March 1983, he joined the Electrical Engineering Department of the California Institute of Technology, Pasadena, as an Assistant Professor, where he has been Professor of electrical engineering since 1993. He has authored a number of papers in IEEE journals and is the author of the book Multirate Systems and Filter Banks (Englewood Cliffs, NJ: Prentice-Hall, 1993). He has written several chapters for various signal processing handbooks. His main research interests are in digital signal processing, multirate systems, wavelet transforms, and signal processing for digital communications.

Dr. Vaidyanathan served as Vice-Chairman of the Technical Program committee for the 1983 IEEE International Symposium on Circuits and Systems and as the Technical Program Chairman for the 1992 IEEE International Symposium on Circuits and Systems. He was an Associate Editor for the IEEE TRANSACTIONS ON CirCuITS AND Systems from 1985 to 1987 and is currently an Associate Editor for the journal IEEE SIGNAL PROCESSING LETTERS, and a Consulting Editor for the journal Applied and Computational Harmonic Analysis. In 1998, he was a Guest Editor for special issues of the IEEE TRANSACTIONS ON SigNAL PROCESSING and the IEEE TRANSACTIONS ON CIRCUITS AND SYSTEMS II, on the topics of filter banks, wavelets, and subband coders. He was a recipient of the Award for Excellence in Teaching at the California Institute of Technology for the years 1983-1984, 1992-1993, and 1993-1994. He also received the NSF's Presidential Young Investigator award in 1986. In 1989, he received the IEEE ASSP Senior Award for his paper on multirate perfect-reconstruction filter banks. In 1990, he was recipient of the S. K. Mitra Memorial Award from the Institute of Electronics and Telecommunications Engineers (IETE), India, for his joint paper in the IETE journal. He was also the coauthor of a paper on linear-phase perfect reconstruction filter banks in the IEEE TRANSACTIONS ON SIGNAL PROCESSING, for which the first author (T. Nguyen) received the Young outstanding author award in 1993. He received the 1995 F. E. Terman Award of the American Society for Engineering Education (ASEE), sponsored by Hewlett Packard Company, for his contributions to engineering education, especially the book Multirate Systems and Filter Banks. He has given several plenary talks, including those at the Sampta'01, EUSIPCO'98, SPCOM'95, and Asilomar' 88 conferences on signal processing. He has been chosen a Distinguished Lecturer for the IEEE Signal Processing Society for the year 1996-1997. In 1999, he was chosen to receive the IEEE CAS Society's Golden Jubilee Medal. He is a recepient of the IEEE Signal Processing Society's Technical Achievement Award for 2002. 